POS $\quad$ PROCEEDINGS

\title{
Superhumps in SU Uma Dwarf Novae
}

\author{
I.B. Voloshina*t \\ Sternberg Astronomical Institute, Moscow M.V.Lomonosov State University \\ E-mail: voloshina.ira@gmail.com
}

Dwarf novae belong to the class of cataclysmic variables and represent the close binary systems in the late evolutionary stages. They consist of a white dwarf and a red dwarf which fills its Roche lobe and transfers mass to the white dwarf. The gas stream flows from the secondary to the primary white dwarf forming an accretion disk around it. Dwarf novae undergo outburst in semi-periodic intervals of time, when the brightness increases by $3^{m}$ to $5^{m}$. For most of them, the faint state (or quiescence) is a normal state. There are 3 types of dwarf novae: U Gem, Z Cam and SU UMa. The last ones have orbital periods about $80-180$ min, mass of the secondary 3 times less or even more than white dwarf mass. Two types of outbursts are observed in these systems: frequent normal outbursts and superoutbursts which last longer and rise to slightly higher luminosities. During superoutburst they exhibit so-called "superhumps", — brightness increase on the small part of the light curve repeated with the period a few percents longer than the orbital one and amplitude of $\sim 0.1^{m}-0.3^{m}$. Superhumps are the most striking phenomenon which exhibited SU UMa dwarf novae and make them interesting and promising objects for study. According to the tidal- thermal model (Osaki, 1996) superhumps are due to gravitational disturbances from the secondary. These disturbances became most effective then the matter of accretion disk reach the 3:1 resonance of the orbital motion of the secondary. The beating of the orbital and precessional periods cause periodic variations, identified as superhumps. Positive and negative superhumps are described. The evolution of superhumps is considered together with the changes of superhump period during the outburst.The observational properties of SU UMa stars are collected by photometric observations of various systems. Criteria for classification of WZ Sge dwarf novae (sub-type of SU UMa stars) are summarized. Rebrightenings or echo outbursts are one of the most striking characteristics of WZ Sge objects.

Accretion Processes in Cosmic Sources - APCS2016-

5-10 September 2016,

Saint Petersburg, Russia

*Speaker.

${ }^{\dagger}$ A footnote may follow. 


\section{Introduction}

Dwarf novae belong to the class of cataclysmic variables and represent the close binary systems in the late evolutionary stages. They consist of a white dwarf and a red dwarf which fills its Roche lobe and transfers mass to the white dwarf. The gas stream flows from the secondary to the primary white dwarf forming an accretion disk around it. Dwarf novae undergo outburst in semi-periodic intervals of time, when the brightness increases by $3^{m}$ to $5^{m}$. For most of them, the faint state (quiescence) is their normal state. During quiescence the disk has a low viscosity and accumulates mass, remaining cool and faint. When the surface density of the disk reaches a critical value, the viscosity increases and mass flows rapidly through the disk to the white dwarf, releasing gravitational energy. The disk became hot and luminous causing the dwarf nova outburst.

\section{Classification of dwarf novae}

There are 3 types of dwarf novae: U Gem, Z Cam, and SU UMa.

U Gem - the classical dwarf novae, outbursts with large amplitude of 4-5 ${ }^{m}$ (a factor of $40-100$ in intensity) and typical cycle from weeks to years.

Z Cam - exhibit long standstills at the mean light level, $1^{m}$ below maximum, these periods of constant light may last for many months, their length varies considerably,

- in standstill they resemble the nova-like systems which never show outbursts.

SU UMa - have orbital periods - about 80-180 min,

- mass of the secondary 3 times less or even more than white dwarf mass,

- two types of outbursts: frequent normal and less often superoutburst,

- they exhibit so-called "superhumps", — brightness increase on the small part of the light curve repeated with the period a few percents longer than the orbital one with amplitudes $\sim 0.1-0.3^{m}$. Superoutbursts last longer and rise to slightly higher luminosities.

SU UMa stars in turn are subdivided into two groups:

WZ Sge-type - unusually high amplitudes $\left(8^{m}\right)$,

- rare outbursts (once a decade),

- the shortest $P_{\text {orb }}$ among DNe ( 80-90 minutes),

- rebrightening, - complex post-superoutburst rise of brightness or echo outbursts,

- absence of normal outbursts (defining characteristic of WZ Sge stars, have been recorded in a few systems).

ER UMa-type - with extremely short interval between superoutbursts $\left(20-50^{d}\right)$,

- from one third to one half of the cycle takes the superoutburst,

- normal outbursts occur every 4 days.

About 10 WZ Sge-type dwarf nova was discovered in 2014, 100 WZ Sge-type stars are known [10], 4 eclipsing systems between them. 


\section{Statistics for WZ Sge stars}

The properties of this class of stars were described in details by Kato in his fine review [10]. For majority of WZ Sge-objects they were defined as a following:

1. the $P_{\text {orb }}$ is shorter than $0.06^{d}$ and lies in the range $0.0553-0.0592^{d}$ (for $50 \%$ stars),

2. the outburst amplitudes larger than $7^{m}$, the largest one $\left(9.5^{m}\right)$ - in SSS J224739,

3. the recurrence time is shorter than $23-33 \mathrm{yr}$, the median value is $11.5 \mathrm{yr}$, the shortest known interval $\left(450^{d}\right)$ - in AL Com.

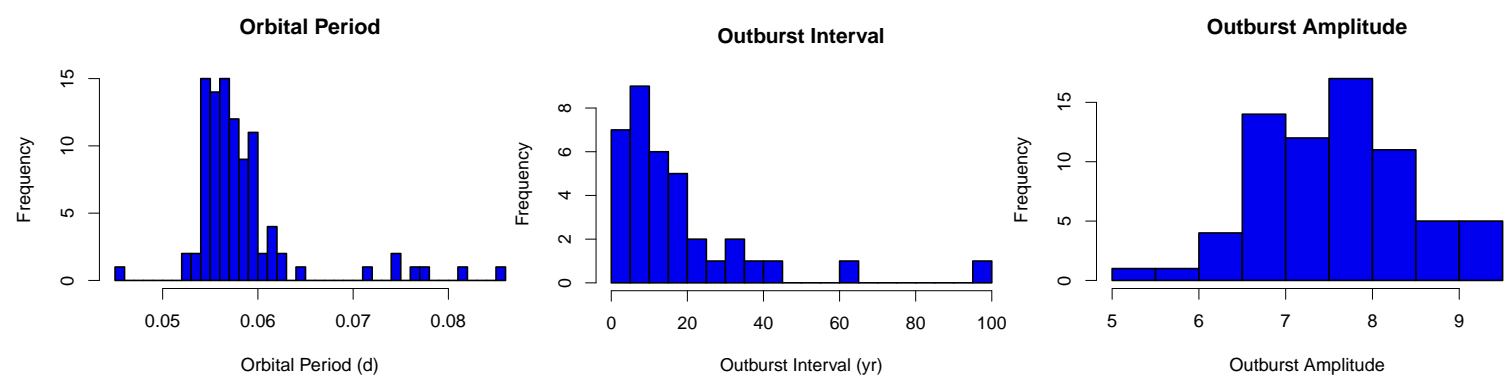

Figure 1: Orbital periods, outburst intervals and amplitudes (left to right) of WZ Sge-objects, according to $[10]$.

\section{Superhumps}

Superhumps are the phenomenon typical for the SU UMa stars. The shape of the superhumps depends on the outburst phase and could changed from one system to another, the period of superhumps changed during the outburst. The evolution of superhumps passes through the next 3 main stages stages according Kato et al.(2009):

stage A early superhumps with more long period of superhumps,

stage B intermediate stage with systematically varying period of superhumps and the last one,

stage $\mathbf{C}$ with short stable periods.

Early superhumps, observed in the beginning of the outburst, have a double hump wave and their periods are very close to the orbital ones (Kato, 2002). They were detected in $63 \%$ of the studied WZ Sge-type dwarf novae [10] with amplitude in average larger than 0.02 mag.

The stage of "ordinary superhumps" changes the "early superhumps" stage. They have a typical one-hump wave and the largest amplitude of periodicity. The period of ordinary superhumps usually is a few percent longer than the orbital period. The ordinary superhumps can be explained by the thermal tidal instability model of an accretion disk (Osaki,1989). The presence of the tidal 3:1 resonance in the disk (with the radius smaller than the 2:1 resonance radius) results in the formation of an eccentric outer ring undergoing apsidal precession with a period appreciably longer than the orbital one. A final stage of superoutburst is characterized by "late superhumps" with 
smaller amplitude and a longer period. The late superhumps appear several days after the rapid decline from the plateau in the light curve of a superoutburst and may continue for several hundred cycles after the end of the superoutburst. According to Kato (2008), the late superhumps originate in the precessing eccentric disk near the tidal truncation. The eccentric disk slowly expands during the superoutburst decline and finally reaches the tidal truncation, where the period is stabilized. In WZ Sge-type dwarf novae stage $\mathrm{C}$ is usually not present.

a).

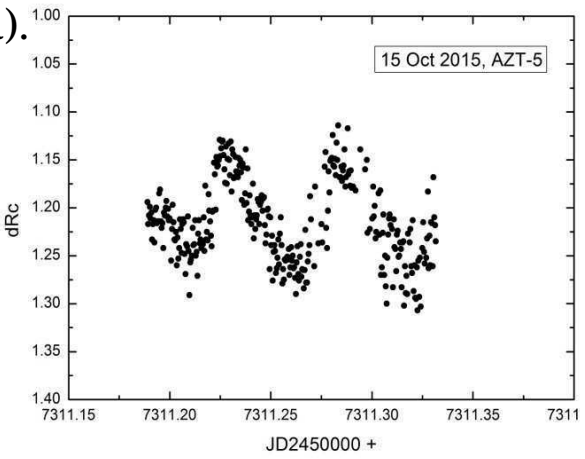

c).

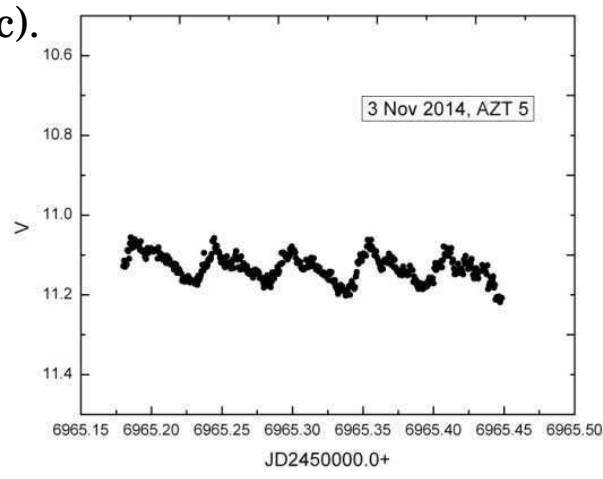

b).

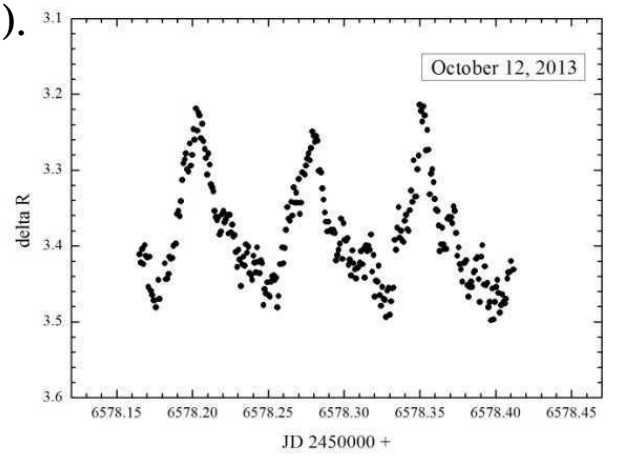

d).

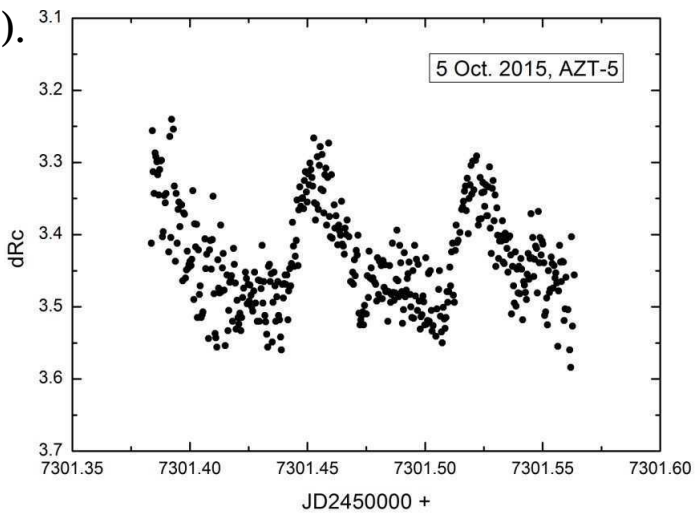

e).

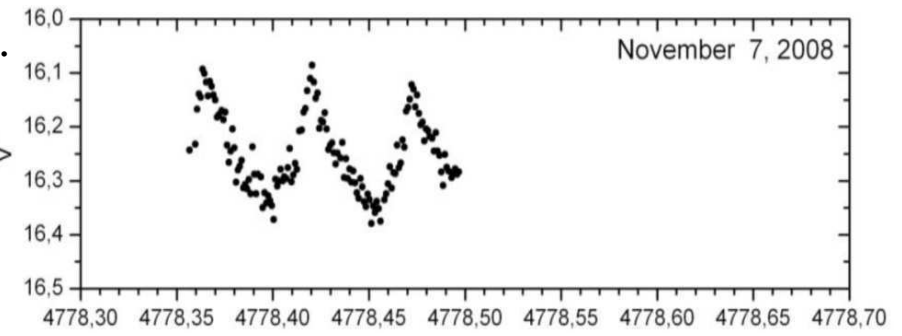

Figure 2: Variety of superhumps in the light curves of different SU UMa stars: a). J1457, $P_{\text {orb }}=0^{d} .05898$; b). AW Sge, $P_{\text {orb }}=0^{d} .0724 ;$ c). J2138, $P_{\text {orb }}=0^{d} .05456$; d). PU Per; e). UW Tri, $P_{\text {orb }}=0^{d} .05334$.

According to the tidal-thermal model (Osaki, 1996) superhumps are due to gravitational disturbances from the secondary. These disturbances become most effective when the matter of accretion disk comes to 3:1 resonance with orbital motion of the secondary. The beating of the orbital and precessional periods cause periodic variations, identified as superhumps. So the superhumps period $P_{s h}$ is related to the precession period $P_{\text {prec }}$ and orbital period $P_{o r b}$ : 


$$
\frac{1}{P_{\text {sh }}}=\frac{1}{P_{\text {orb }}}-\frac{1}{P_{\text {prec }}}
$$

and the fractional superhump excess, introduced by Kato (2014a), in the frequency unit:

$$
\epsilon=1-\frac{P_{\text {orb }}}{P_{\text {sh }}}
$$

The fractional superhump excess is a direct measure of the precession rate.

This model is supported by numerical simulations (Bisikalo et al., 2005; Kaigorodov et al., 2006; Smith et al., 2007).
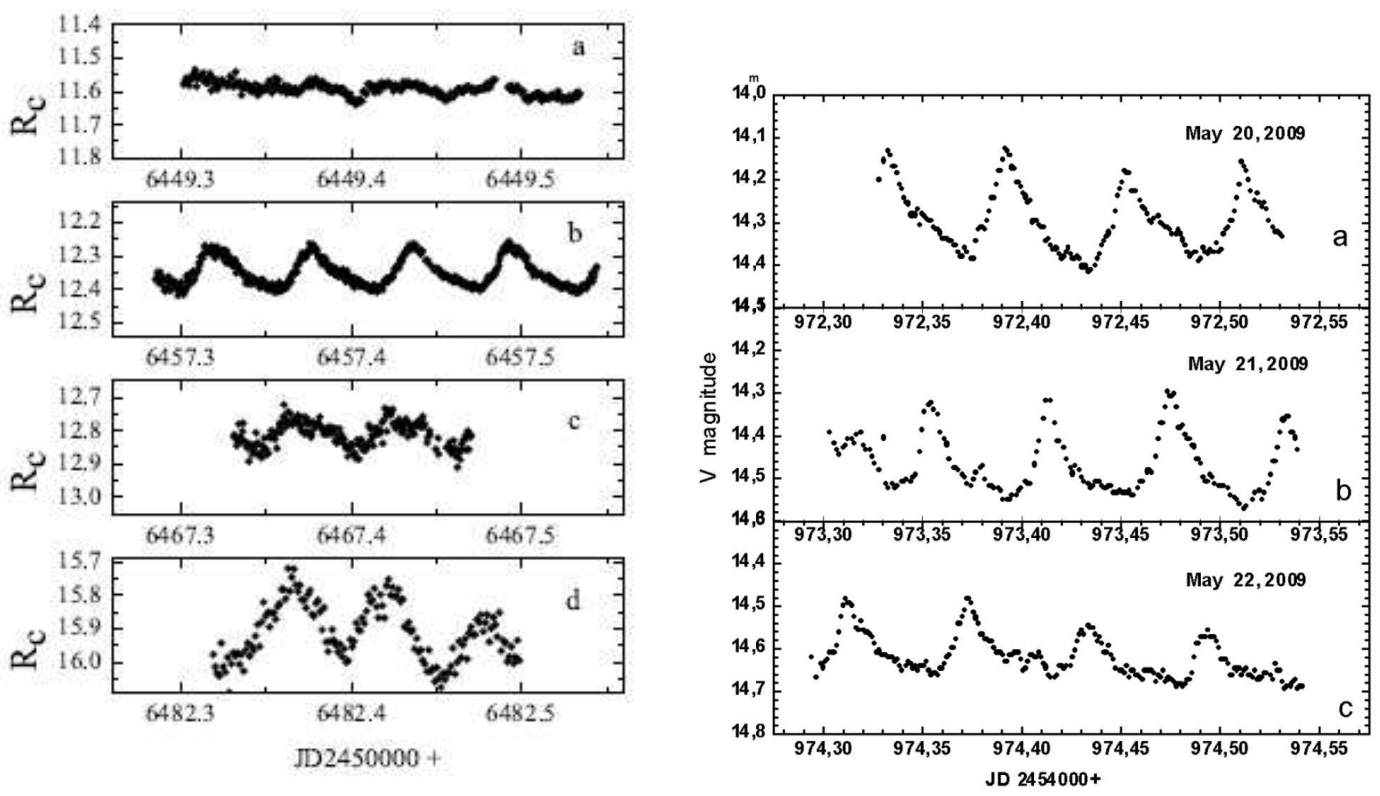

Figure 3: Left panels: Evolution of superhumps in dwarf nova J1915: a - beginning of stage A superhumps; $\mathrm{b}$ - stage B superhumps; $\mathrm{c}$ - transition from B to C stage; $\mathrm{d}$ - post-fading stage superhumps (scale is the same). Right panels: Superhumps in dwarf nova KV Dra, $P_{\text {orb }}=0^{d} .05876$, amplitude of superhumps decreases with time, their profile becames less steep. Decrease of superhumps which is clearly seen in the lowest light curve evidence that a new stage is approaching.

\subsection{Negative superhumps}

It was found that not all SU UMa stars showed on their light curves "positive superhumps". Some of them demonstrate variations with the period, shorter than the orbital one. Such variations called "negative superhumps" (Ringwald et al., 2012). The first detection of negative superhumps during the superoutburst of SU UMa-type DNe was done for ER UMa superoutburst in 2011. Later they were also reported during normal outbursts and quiescence with an amplitude $\sim 0.5-1.0^{m}$. Dwarf nova MN Dra displayed negative superhumps between the superoutbursts (Pavlenko et al., 2010). The existence of negative superhumps during a superoutburst was reported for other SU UMa dwarf novae: V1504 Cyg and V344 Lyr (Osaki \& Kato, 2013).

The origin of negative superhumps is usually considered as a result of retrograde precession in a tilted accretion disk [12]. When the disk is tilted, the hot spot is formed at the inner part of 


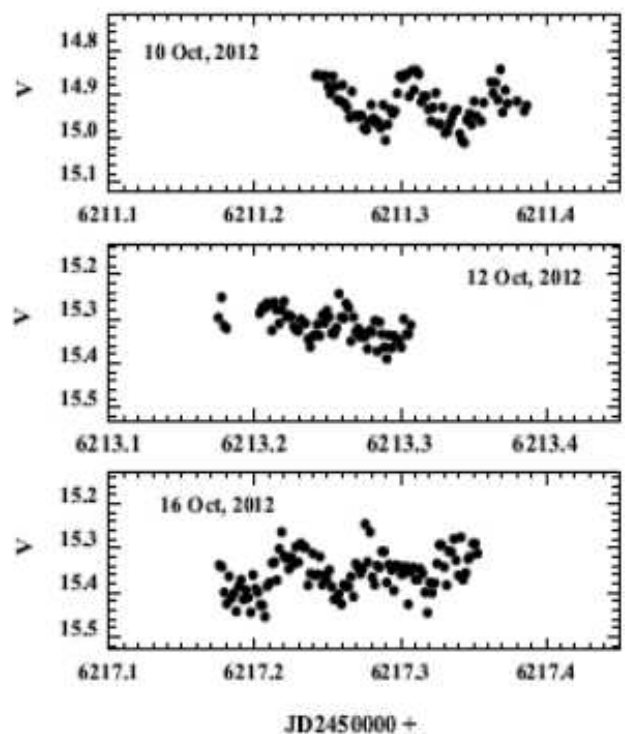

Figure 4: Stage B superhumps observed in the dwarf novae J2051.

the disk, not at the edge of the disk. Since the energy of the hot spot comes from the release of gravitational energy, such a change in the location of the hot spot causes a variation in the amount of released energy, namely, the luminosity of the hot spot. Combined with the retrograde precession, this effect can explain the negative superhumps.

Superoutbursts occur more regularly than the normal outbursts. Several normal outbursts occurred between two superoutbursts (see fig. 5). The peak magnitude of a normal outburst becomes brighter and the cycle length becomes longer as the next superoutburst approaches.

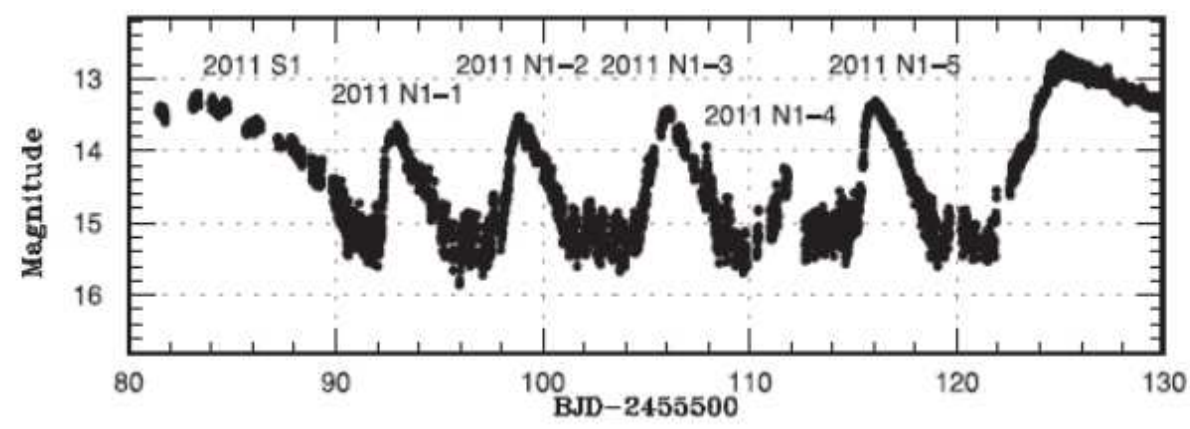

Figure 5: ER UMa light curve.

For explanation of such behaviour of SU UMa dwarf novae, the thermal tidal instability (TTI) model was suggested (Osaki, 1989). In this model, systems with a small mass ratio $\left(M_{2} / M_{1}=\right.$ $q \leqslant 0.25$ ) enable the disk radius to reach the $3: 1$ resonance of the orbital motion of the secondary. In normal outbursts, the disk material only partly accretes to the inner region. The radius of the disk becomes gradually larger after experiencing a normal outburst. When the disk radius reaches 
the 3:1 resonance one, the eccentric instability is excited. The increased turbulence in the disk increases the mass-transfer rate in the disk and a long, bright superoutburst is triggered (Osaki 1989).

Stage A superhumps are currently understood to reflect the dynamical precession rate of the disk at the radius of 3:1 resonance, stage $\mathbf{B}$ is considered to have a smaller precession rate due to the pressure effect, which produces retrograde precession, in the disk, the origin of stage $\mathbf{C}$ superhumps and why stage $B-C$ transition suddenly occurs are unsolved problems.
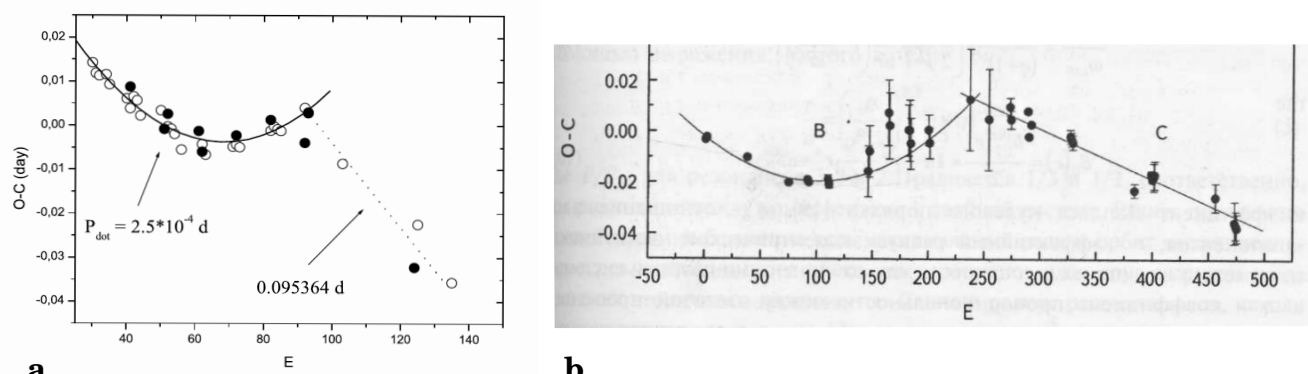

b
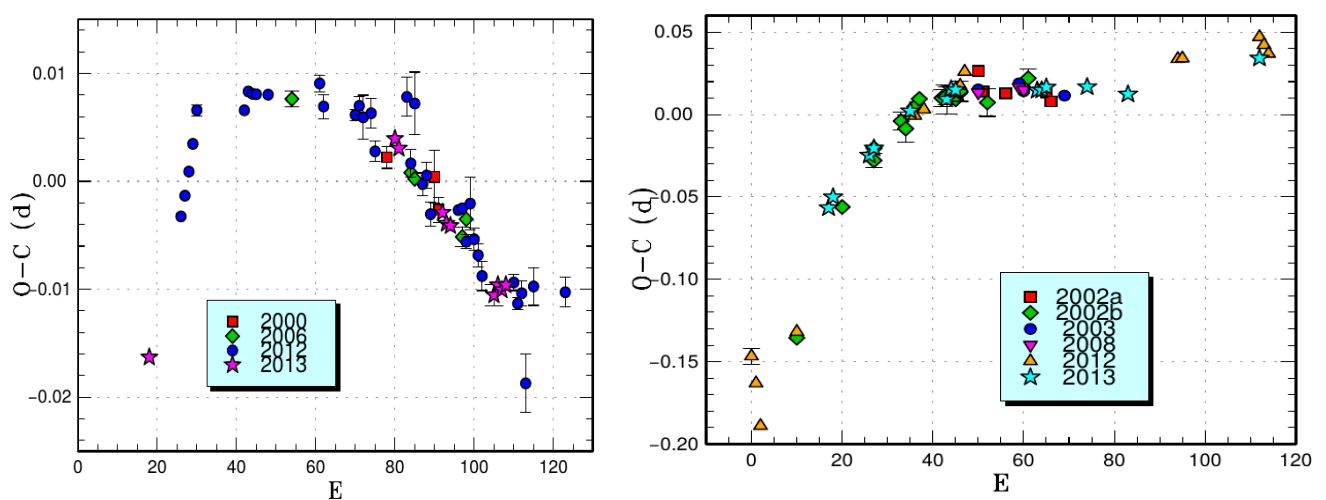

c

d

Figure 6: The O-C diagrams of superhumps in SU UMa stars: a). dwarf nova J1625, b). dwarf nova J2138, c). AW Sge and d). dwarf nova MN Dra. Figures c). and d). are taken from [Kato et al., 2014a].

\section{Phenomenon of rebrightenings}

Rebrightenings or echo outbursts are one of most striking characteristics of WZ Sge objects. Imada et al. (2006) suggested to classify all detected rebrightenings in following 4 types:

- type-A outbursts (long-duration rebrightening),

- type-B outbursts (multiple rebrightenings),

- type-C outbursts (single rebrightening) and

- type-D outbursts (no rebrightening). 
Type-A outbursts and type-D outbursts tend to occur in objects with short $P_{\text {orb }}$. Type-C outbursts are usually seen in objects with longer $P_{o r b}$. For Type B the numbers of observed rebrightenings can vary. Later Kato et al. (2014) suggested to add another type, - Type-E. It was done after the detection of two objects with double superoutbursts: SSS J122221 and OT J184228.

It is not known yet whether the dwarf nova shows the same type of rebrightenings during every outburst.

The main properties of WZ Sge-type objects with multiple rebrightenings were discribed by Meyer, Meyer-Hofmeister (2015). The study of type-B objects shows thet the number of rebrightening ranges from 2 to 11 . Their amplitudes are correlated with intervals of rebrightenings changing approximately from 2 to 10 days. Average amplitude of rebrightenings range from 1.6 to 3.7 mag.
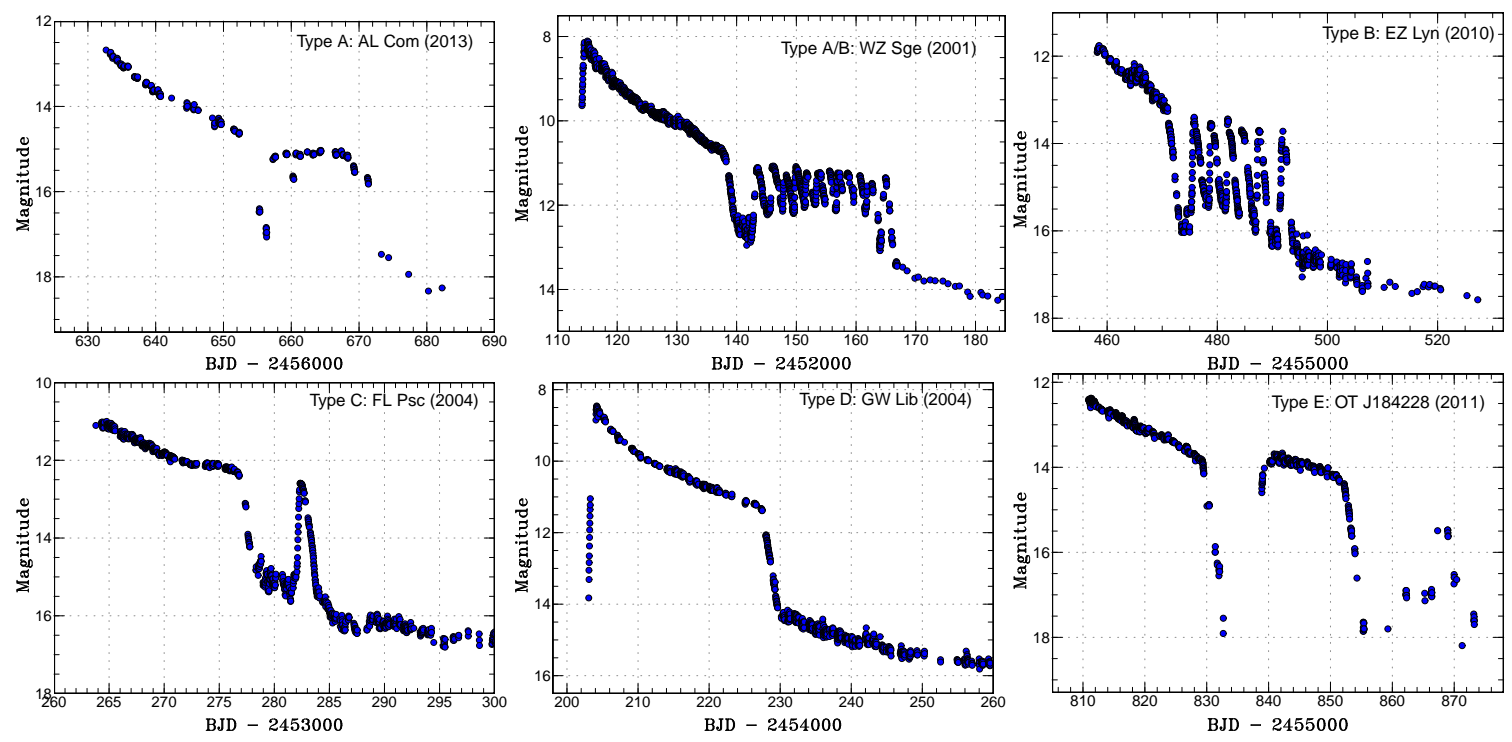

Figure 7: Examples of different types of rebrightening according to Kato et al. (2015).

\section{Summary}

1. Superhumps are the striking phenomenon which exhibited SU UMa dwarf novae and make them interesting and promising objects for study,

2. the presence of early superhumps and long or multiple rebrightenings are the best distinguishing properties WZ Sge-type of dwarf novae,

3. the observational properties of SU Uma stars are collected in detailed Surveys of Period Variations of Superhumps in SU UMa-Type Dwarf Novae initiated and worked out by T.Kato (8 surveys at all and one more in preparation).

4. Despite these papers, many aspects of SU UMa dwarf novae study still wait for the further investigations, particularly in theoretical field. The TTI model fails to reproduce the amplitudes of superhumps (Smak, 2009). In his new interpretation of superhumps, periodically 
variable irradiation of the secondary component results in a strongly modulated mass outflow. Superhumps are then due to enhanced dissipation of the kinetic energy of the stream (Smak, 2016). But also this model has difficulties.

Criteria for classification of WZ Sge dwarf novae could be summarized in the following way:

- the amplitude of outburst is close to $8^{m}$;

- the superoutbursts of WZ Sge-type objects are very rare in comparison with other SU UMa dwarf novae;

- detection of "early superhumps" which are the unique feature of WZ Sge-type objects, early superhumps show double-wave per a period with $\mathrm{P}_{\text {early }}$;

- stage $\mathrm{C}$ is usually absent;

- the typical value of $\epsilon$ for WZ Sge objects is $\epsilon=1-P_{\text {orb }} / P_{\text {sh }} \approx 0.03$

\section{Acknowledgements}

The author is grateful to Dr. Valerian Sementsov for useful discussion. This study has been supported via grant of the Program of Leading Scientific Schools of Russian Federation (7065.2016.2).

\section{References}

[1] Bisikalo D. V., Kaigorodov, P. V., Boyarchuk, A. A., Kuznetsov, O. A., The Possible Nature of Dips in the Light Curves of Semidetached Binaries with Stationary Disks 2005, ARep 49, 701

[2] Imada A., Kubota, K., Kato, T., et al., Discovery of a New Dwarf Nova TSSJ022216.4=412259.9: WZ Sge type Dwarf Nova Breaking the shortest Superhump Period Record 2006, PASJ, 58, L23

[3] Kaigorodov, P. V., Bisikalo, D. V., Kuznetsov, O. A., Boyarchuk, A. A., Superhumps in binary systems and their connection to precessional spiral density waveso 2006, ARep 50, 537

[4] Kato, T., On the Origin of Early Superhumps in WZ Sge-type stars 2002, PASJ, 54, L11

[5] Kato, T., Survey of Period Variations of Superhumps in SU UMa-Type Dwarf Novae. III. The Third Year (2010-2011) 2012, PASJ, 64, 21

[6] Kato, T., Maehara, H., \& Monard, B., Late Superhumps in WZ Sge-Type Dwarf Novae 2008, PASJ, 60, L23

[7] Kato, T., et al., Survey of Period Variations of Superhumps in SU UMa-Type Dwarf Novae. IV. The Fourth Year (2011-2012) 2013, PASJ, 65, 23

[8] Kato, T. , Survey of period variations of superhumps in SU UMa-type dwarf novae. V. The fifth year (2012-2013) 2014, PASJ, 66, 30

[9] Kato, T., Survey of period variations of superhumps in SU UMa-type dwarf novae. VI. The sixth year (2013-2014) 2014, PASJ, 66, 90

[10] Kato, T., et al., WZ Sge-Type Dwarf Novae 2015, PASJ, 67, 105 
[11] Meyer, F., Meyer-Hofmeister, E., SU UMa stars: Rebritenings after Superoutburst 2015, PASJ, 67, 52

[12] Montgomery, M. M., Numerical Simulations of Naturally Tilted, Retrogradely Precessing, Nodal Superhumping Accretion Disks 2012, Astrophys. J. Lett., 745, L25

[13] Montgomery, M. M., Voloshina, I., \& Goel, A.,Photometric Observations and Numerical Modeling of SDSS J162520.29+120308.7, an SU UMa in the CV Period Gap 2016, New Astronomy, 42, 78

[14] Osaki, Y.,A model for the superoutburst phenomenon of SU Ursae MAjoris stars 1989, PASP, 41, 1005

[15] Osaki, Y.,Dwarf-Nova Outbursts 1996, PASP, 108, 39

[16] Osaki,Y., Kato, T., The Cause of the Superoutburst in SU UMa Stars is Finally Revealed by Kepler Light Curve of V1504 Cygni 2013, PASJ, 65, 50

[17] Pavlenko, E., Kato, T., Andreev, M., Sklyanov, A., Zubareva, A., Samsonov, D., Voloshina, I., et al.,MN DraÜIn-the-Gap Dwarf Nova With Negative Superhumps 2010, 17th European White Dwarf Workshop. AIP Conf. Proc., 1273, 320

[18] Smak, J., On the Amplitudes of Superhumps 2009, Acta Astronomica, 59, 103

[19] Smak, J., Superhumps and their Evolution during Superoutbursts 2016, Acta Astronomica, 66, 75

[20] Smith, A .J., Haswell, C. A., Murray, J. R., et al., Comprehensive simulations of superhumps 2007 , MNRAS, 378, 785

[21] Ringwald, F. A., Velasco, K., Roveto, J. J., Meyers, M. E.,The orbital period and negative superhumps of the nova-like cataclysmic variable V378 Pegasi 2012, New Astronomy, 17, 433

[22] Voloshina, I., \& Metlov, V., Study of Photometric Variability of Selected SU UMa Dwarf Novae 2015, Acta Polytechnica CTU Proceedings, 2, 161 